\title{
RESEARCH
}

Open Access

\section{Social brain activation during mentalizing in a large autism cohort: the Longitudinal European Autism Project}

Carolin Moessnang $^{1 *}$ D , Sarah Baumeister ${ }^{2}$, Julian Tillmann ${ }^{3,4}$, David Goyard $^{5}$, Tony Charman ${ }^{3}$, Sara Ambrosino ${ }^{6}$, Simon Baron-Cohen 7 , Christian Beckmann ${ }^{8,9}$, Sven Bölte ${ }^{10,11}$, Carsten Bours ${ }^{8,9}$, Daisy Crawley ${ }^{12}$, Flavio Dell'Acqua ${ }^{12,13}$, Sarah Durston ${ }^{6}$, Christine Ecker ${ }^{14}$, Vincent Frouin ${ }^{5}$, Hannah Hayward ${ }^{12}$, Rosemary Holt ${ }^{7}$, Mark Johnson ${ }^{15}$, Emily Jones ${ }^{15}$, Meng-Chuan Lai ${ }^{7,16,17}$, Michael V. Lombardo ${ }^{7,18}$, Luke Mason ${ }^{15}$, Marianne Oldenhinkel ${ }^{8,9}$, Antonio Persico ${ }^{19,20}$, Antonia San José Cáceres ${ }^{12}$, Will Spooren ${ }^{21}$, Eva Loth ${ }^{12,13}$, Declan G. M. Murphy ${ }^{12,13}$, Jan K. Buitelaar ${ }^{8,9,22}$, Tobias Banaschewski ${ }^{2}$, Daniel Brandeis ${ }^{2,23,24}$, Heike Tost ${ }^{1}$, Andreas Meyer-Lindenberg ${ }^{1}$ and the EU-AIMS LEAP group

\begin{abstract}
Background: Autism spectrum disorder (ASD) is a neurodevelopmental condition with key deficits in social functioning. It is widely assumed that the biological underpinnings of social impairment are neurofunctional alterations in the "social brain," a neural circuitry involved in inferring the mental state of a social partner. However, previous evidence comes from small-scale studies and findings have been mixed. We therefore carried out the todate largest study on neural correlates of mentalizing in ASD.

Methods: As part of the Longitudinal European Autism Project, we performed functional magnetic resonance imaging at six European sites in a large, well-powered, and deeply phenotyped sample of individuals with ASD ( $N=$ 205) and typically developing (TD) individuals $(N=189)$ aged 6 to 30 years. We presented an animated shapes task to assess and comprehensively characterize social brain activation during mentalizing. We tested for effects of age, diagnosis, and their association with symptom measures, including a continuous measure of autistic traits.

Results: We observed robust effects of task. Within the ASD sample, autistic traits were moderately associated with functional activation in one of the key regions of the social brain, the dorsomedial prefrontal cortex. However, there were no significant effects of diagnosis on task performance and no effects of age and diagnosis on social brain responses. Besides a lack of mean group differences, our data provide no evidence for meaningful differences in the distribution of brain response measures. Extensive control analyses suggest that the lack of case-control differences was not due to a variety of potential confounders.

(Continued on next page)
\end{abstract}

\footnotetext{
* Correspondence: carolin.moessnang@zi-mannheim.de

${ }^{1}$ Department of Psychiatry and Psychotherapy, Central Institute of Mental Health, Medical Faculty Mannheim / University of Heidelberg, Mannheim, Germany

Full list of author information is available at the end of the article
}

(c) The Author(s). 2020 Open Access This article is distributed under the terms of the Creative Commons Attribution 4.0 International License (http://creativecommons.org/licenses/by/4.0/), which permits unrestricted use, distribution, and reproduction in any medium, provided you give appropriate credit to the original author(s) and the source, provide a link to the Creative Commons license, and indicate if changes were made. The Creative Commons Public Domain Dedication waiver (http://creativecommons.org/publicdomain/zero/1.0/) applies to the data made available in this article, unless otherwise stated. 


\begin{abstract}
(Continued from previous page)
Conclusions: Contrary to prior reports, this large-scale study does not support the assumption that altered social brain activation during mentalizing forms a common neural marker of ASD, at least with the paradigm we employed. Yet, autistic individuals show socio-behavioral deficits. Our work therefore highlights the need to interrogate social brain function with other brain measures, such as connectivity and network-based approaches, using other paradigms, or applying complementary analysis approaches to assess individual differences in this heterogeneous condition.
\end{abstract}

Keywords: Autism, Autism spectrum disorder, Social brain, fMRl, Mentalizing, Theory of mind, Animated shapes, Development, Multi-site

\section{Background}

Atypicalities in social communication and interaction are a hallmark of autism spectrum disorder (ASD), a neurodevelopmental condition with onset in early childhood. The ability to recognize the mental state of a social partner, also referred to as theory of mind (ToM), has long been posited to be altered in autism in the "mind-blindness" account of ASD [1]. These deficits are suggested to be exacerbated during on-line mentalizing, e.g., during the instantaneous use of ToM in real-life social interactions, while impairments in explicit mentalizing, such as the instructed reasoning about mental states, can be related to (and compensated by) executive function abilities [2-6].

The mind-blindness account has stimulated a line of imaging research to explore the potential neurobiological underpinnings of mentalizing, and of social cognition in general, in ASD. In a seminal paper, Castelli and colleagues [7] presented short animations of geometric shapes whose movement patterns prompted mental state attribution (e.g., a triangle mocking another triangle [8]). In contrast to false-belief tasks, where the mental state of a social agent can be deduced by logical inference based on a given scenario, the animated shapes draw upon our irresistible tendency to attribute mental states-even to non-living beings devoid of facial or other human-like cues-in the very moment of watching $[8,9]$. The authors found reduced activation in a sample of 10 adult ASD participants in regions of the so-called social brain, a neural network that is commonly activated in tasks involving mentalizing, especially the posterior superior temporal sulcus (pSTS), extending into the temporo-parietal junction (TPJ), and the dorsomedial prefrontal cortex (dmPFC [10]). This hypoactivation was paralleled by less accurate verbal descriptions of ToM animations. The authors suggested that mentalizing deficits in ASD might originate from a failure to adequately extract and process social meaning from sensory input. If validated in large samples, social brain responses to animated shapes could be exploited for biomarkers related to diagnosis, stratification, and/or treatment prognosis in ASD [11].
Numerous imaging studies on social cognition in ASD have since been performed. Here, the use of a wide variety of tasks and analysis procedures across different samples has resulted in a heterogeneous picture of neurofunctional alterations in ASD that are at times contradictory to the findings by Castelli et al. [7]. For instance, among those studies that employed an animated shapes task, methodological differences include (a) variations in ASD sample composition (e.g., 10 adults [mean age $33 \pm$ 7.6 years, sex not specified] in [7]; 12 adolescents/adults [15 to 35 years, 2 females] in [12]; 13 adolescents [10 to 16 years, 2 females] in [13]; 17 adolescents/adults [13 to 23 years, 4 females] in [14]), (b) differences in imaging data analysis (e.g., different preprocessing routines with smoothing kernels ranging from $4.5 \mathrm{~mm}$ in [14] to 16 $\mathrm{mm}$ in [7], significance assessment on the whole-brain level $[7,12,13]$ and/or within regions of interest $[13$, $14]$ ), and even (c) differences in imaging modality (positron emission imaging in [7], fMRI in [12-14]). These and other studies on social cognition have produced mixed conclusions on ASD-related effects, ranging from reduced to excess activation in different brain regions within or outside the social brain, or no effect at all. In order to detect converging evidence, systematic metaanalyses have been performed but results vary with the studies included (e.g., [15-19]). Thus, while several candidate regions such as the medial prefrontal cortex, pSTS, amygdala, insula, fusiform face area, and inferior frontal gyrus (IFG) have been highlighted in the context of altered social information processing in ASD, the overall picture remains inconclusive.

An important source of variance in the ASD imaging literature is age, which needs to be addressed explicitly when studying developmental samples. The literature points to higher activation of frontal areas during adolescence compared to adulthood in typically developing individuals, which might reflect ongoing prefrontal maturation and less efficient inhibitory control in adolescence $[20,21]$. However, this effect is not specific for social cognition and more research is warranted to address the effect of age on social brain function and its interaction with the autistic condition. 
More recently, large-scale multicenter studies have been launched using more representative samples with higher statistical power. These have so far been limited to resting state imaging data $[22,23]$ which do not allow to draw conclusions on time-locked functional responses to external stimuli or task demands.

We have therefore extended previous large-scale efforts to include brain activation measures in the Longitudinal European Autism Project (LEAP [24]) where a well-powered, representative, and deeply phenotyped sample of participants with ASD and typically developing (TD) individuals has been characterized from childhood to adulthood. This unique cohort allows to address several key limitations of earlier studies, such as small sample size and low statistical power [25], restriction to specific age ranges, exclusion of the broader autism phenotype involving comorbidities, and limited clinical, psychological, and biological characterization for stratification analysis. In the current study, our aim was to discover and validate neurofunctional markers of social cognition alterations in ASD as a first step for biomarker discovery. The animated shapes task was chosen as one of four neurocognitive paradigms in this large study, given the promising findings in earlier reports that suggest high construct validity for on-line mentalizing deficits in ASD [7, 26, 27], and due to its good applicability across age ranges and intellectual abilities. Here, we used an adapted version of the task [27] that was recently shown to have reproducible effects on functional activation [28] and to be sensitive for autism-related traits [29]. Functional responses were comprehensively assessed as changes in brain activation and related to age and clinical status. We also undertook a dimensional analysis approach to investigate the influence of autismrelated traits on social brain development. We expected individuals with ASD or with higher autism-related traits to show reduced regional activation in key areas of the social brain in response to the animated shapes $[7,13$, 14]. Regarding the effect of age independent from diagnostic group, we expected younger participants to show higher frontal activation, reflecting a stronger involvement of areas implicated in executive control [20, 21].

\section{Methods}

\section{Sample}

Participants were part of EU-AIMS LEAP, a large multicenter European initiative aimed at the identification of biomarkers in ASD [24]. The study comprises 437 individuals with ASD and 300 TD individuals, both males and females, aged between 6 and 30 years. Participants underwent comprehensive clinical, cognitive, and MRI assessment at one of the following six centers: Institute of Psychiatry, Psychology and Neuroscience, King's College London, UK; Autism Research Centre, University of
Cambridge, UK; Radboud University Nijmegen Medical Centre, the Netherlands; University Medical Centre Utrecht, the Netherlands; Central Institute of Mental Health, Mannheim, Germany; and University Campus Bio-Medico of Rome, Italy. The study was approved by the local ethical committees of participating centers, and written informed consent was obtained from all participants or their legal guardians (for participants $<18$ years). Individuals with ASD were included in the ASD sample based on an existing clinical diagnosis according to DSM-IV [30], DSM-IV-TR [31], DSM-5 [32], or ICD10 [33]. Given the better accuracy of clinical judgments [34], individuals with ASD were not excluded if they did not reach the cutoff scores on the Autism Diagnostic Observation Schedule (ADOS [35]) or the Autism Diagnostic Interview-Revised (ADI-R [36]) during clinical characterization. For further details about inclusion and exclusion criteria and for a comprehensive clinical characterization of the LEAP cohort, we refer to Charman et al. [37]. For further details on the study design, we refer to Loth et al. [24]. In the present study, we selected all participants with an IQ $>75$ for whom a structural and the task fMRI scan were available $\left(n_{\mathrm{TD}}=231\right.$, $\left.n_{\mathrm{ASD}}=273\right)$. Participants with structural anomalies $(n=$ $8)$, an incomplete task fMRI scan $(n=5)$, excessive head motion during the task fMRI scan $(n=74$; defined as more than $20 \%$ of frames with a framewise displacement (FD) $>0.5 \mathrm{~mm}$; Jenkinson et al. [38]), incomplete information $(n=11)$, and/or corrupted datasets due to technical failure $(n=16)$ were excluded. This resulted in the inclusion of 394 individuals, 205 individuals with ASD and 189 TD individuals, in our analyses (see Table 1 for an overview over key descriptive variables for the full sample, and Additional file 1: Table S1 for a sample description split by age group). Standard operation and quality control procedures are detailed in Additional file 1.

\section{Power analysis}

We performed a power analysis for our sample using GPower [42] to assess statistical sensitivity to detect an effect of group (TD vs. ASD) or an effect of age in a single hypothesis test (e.g., single voxel or region of interest) at a type I error rate of $\alpha=.05$ while accounting for covariates of no interest (sex and site, see below; statistical test in GPower: linear multiple regression, fixed model, $R^{2}$ increase; number of tested predictors, 1 ; total number of predictors, 8). Based on this model, the study has a power of $80 \%$ to detect a standardized effect size of $f^{2} \geq .02$ and a power of $95 \%$ to detect a standardized effect size of $f^{2} \geq .03$, with $f^{2} \geq .02$ denoting a small effect and $f^{2} \geq .15$ denoting a medium effect [43]. In addition, with our large sample of $N>400$, this study is less prone to 
Table 1 Sample description

\begin{tabular}{|c|c|c|c|}
\hline & ASD & TD & Statistics: ASD vs. TD \\
\hline Total $n$ & 205 & 189 & \\
\hline \multicolumn{4}{|l|}{ Demographics } \\
\hline Sex (male/female) & $151 / 54$ & $123 / 66$ & $x^{2}(1)=3.42, p=.079$ \\
\hline Age (years) & $17.9 \pm 5.4(7.1-30.6)$ & $18.0 \pm 5.5(7.6-31.0)$ & $t(392)=.32, p=.749$ \\
\hline IQ (full IQ) & $107.1 \pm 14.2(75.6-148.0)$ & $108.5 \pm 12.1(76.8-142.0)$ & $t(390)=1.05, p=.295$ \\
\hline Handedness (right/left/ambidext/unknown) & $146 / 22 / 8 / 29$ & $131 / 16 / 4 / 38$ & $x^{2}(3)=3.66, p=.301$ \\
\hline Medication use (\% of subjects) ${ }^{1}$ & 40.5 & 5.8 & $x^{2}(1)=62.37, p<.001$ \\
\hline \multicolumn{4}{|l|}{ In-scanner performance } \\
\hline Mean framewise displacement (FD; in $\mathrm{mm})^{2}$ & $.14 \pm .08(.03-.47)$ & $.12 \pm .08(.03-.42)$ & $t(392)=1.88, p=.061$ \\
\hline Volumes with FD > 0.5 mm (\%) & $3.55 \pm 4.74(0-19.40)$ & $3.17 \pm 4.33(0-19.73)$ & $t(392)=.82, p=.412$ \\
\hline Signal-to-noise ratio & $9.7 \pm 1.3(6.5-13.8)$ & $9.9 \pm 1.4(6.5-13.8)$ & $t(392)=1.42, p=.155$ \\
\hline Task accuracy & $.81 \pm .13(.33-1.0)$ & $.83 \pm .13(0-1.0)$ & $t(392)=1.30, p=.194$ \\
\hline \multicolumn{4}{|l|}{ Clinical characteristics } \\
\hline \multicolumn{4}{|l|}{$A D I-R^{3}$} \\
\hline Social interaction & $15.5 \pm 6.7(0-29)$ & & \\
\hline Communication & $12.6 \pm 5.6(0-26)$ & & \\
\hline RRB & $4.5 \pm 2.6(0-12)$ & & \\
\hline \multicolumn{4}{|l|}{ ADOS- $2^{4}$} \\
\hline Social affect & $5.7 \pm 2.5(1-10)$ & & \\
\hline RRB & $4.7 \pm 2.6(1-10)$ & & \\
\hline Total & $5.0 \pm 2.6(1-10)$ & & \\
\hline \multicolumn{4}{|l|}{ SRS-2 (parent report) ${ }^{5}$} \\
\hline Raw score & $86.1 \pm 30.6(21-163)$ & $19.9 \pm 14.4(1-74)$ & $t(245)=19.07, p<.001$ \\
\hline$T$ score & $69.2 \pm 12.1(43-90)$ & $45.1 \pm 5.8(37-66)$ & $t(245)=17.54, p<.001$ \\
\hline \multicolumn{4}{|l|}{ DAWBA comorbidities ${ }^{6}$} \\
\hline ADHD symptoms & $1.6 \pm 1.6(0-5)$ & $.2 \pm .7(0-3)$ & $t(211)=7.25, p<.001$ \\
\hline Depression symptoms & $1.1 \pm 1.3(0-5)$ & $.4 \pm .7(0-4)$ & $t(311)=5.66, p<.001$ \\
\hline Anxiety symptoms & $2.5 \pm 1.3(0-5)$ & $1.2 \pm .9(0-4)$ & $t(348)=11.06, p<.001$ \\
\hline
\end{tabular}

Participant characteristics, split by sample and age group. If not otherwise indicated, numbers reflect mean \pm standard deviation, followed by value range (minimum-maximum)

${ }^{1}$ Percentage of participants taking medication prescribed for behavioral or neurological problems. Medication data was available for 132 ASD and 78 TD participants. See Additional file 1: Table S1 more information on medication

${ }^{2}$ Motion was assessed as mean framewise displacement according to [38]

${ }^{3}$ Autism Diagnostic Interview-Revised (ADI-R [36]) scores were computed for reciprocal interaction (social interaction), communication, and restrictive, repetitive stereotyped behaviors and interests (RRB). ADI-R scores reflect historical symptom severity (age 4-5 years) and were available for 197 ASD participants

${ }^{4}$ Autism Diagnostic Observation Schedule 2 (ADOS-2 [35]). Calibrated severity scores [39] were computed for social affect, RRB, and the overall total score. ADOS-2 scores reflect current symptom severity and were available for 198 ASD participants

${ }^{5}$ Total raw and total $T$ score (sex and age normalized) on the Social Responsiveness Scale-2 (SRS-2 [40]). SRS-2 scores were available for 247 participants (i.e., TD adults are excluded from this measure; see Additional file 1 for analyses using self-reported SRS-2 scores). The raw SRS-2 scores were used in our analyses ${ }^{6}$ Comorbid symptoms of ADHD, depression, and anxiety were assessed with the Development and Well-Being Assessment (DAWBA [41]), generating six levels (ordinal scores 0 to 5 ) of prediction of the probability of a disorder $(\sim 0.1 \%, \sim 0.5 \%, \sim 3 \%, \sim 15 \%, \sim 50 \%,>70 \%)$. DAWBA scores were available for 167 ASD and 146 TD participants for depression, 140 ASD and 73 TD participants for ADHD, and 183 ASD and 167 TD participants for anxiety

false positives and overestimation of effect sizes, in particular in the scenario of weak diffuse effects which is typical for task fMRI [44].

\section{Clinical measures}

Participants in the ASD group had an existing clinical diagnosis of ASD according to the DSM-IV-TR/ICD10 or DSM-5 criteria. ASD symptoms were comprehensively assessed using the Autism Diagnostic Interview-Revised (ADI-R [36]) and Autism Diagnostic Observation Schedule 2 (ADOS-2 [35]). In the current sample, $85 \%$ of ASD individuals met the diagnostic threshold on the ADI-R or ADOS-2, while $49 \%$ met the cutoff on both instruments. Individuals who did not reach the cutoff on either scale were included on the basis of careful clinical judgment made by expert 
clinicians in the participating ASD specialist centers. We used the parent-reported total raw score on the Social Responsiveness Scale Second Edition (SRS-2 [40]) as a continuous measure for autism traits across all participants. The SRS-2 allows for the assessment of autism traits across clinical and non-clinical samples and includes 65 questions about autistic behaviors, generating scores ranging from 0 to 195, with higher scores indicating the presence of pronounced traits. This measure was available for all ASD and TD individuals except for TD adults where only the selfreport was assessed. Analyses on SRS-2 scores including TD adults are presented in Additional file 1.

\section{Task}

We assessed functional brain responses during an adapted version of the animated shapes task [27, 29] used in Castelli et al. [7] which was presented as part of a structural and functional imaging battery [24]. The task consisted of short video clips (26 to $48 \mathrm{~s}$ ) featuring two triangles whose movement patterns reflected increasing levels of mental state attribution according to three conditions: (1) no or little mental state attribution for random movement (e.g., floating around), (2) perception of agency and hence of mental states for goaldirected movement (e.g., chasing), and (3) perception of complex mental states involving theory of mind (e.g., cheating). Four videos per condition were presented in a pseudorandomized order, with no more than two animations of the same condition presented in a row. Participants passively watched each video clip. In the subsequent response phase ( $5 \mathrm{~s}$ ), participants were asked to categorize the animation to one of the three conditions by selecting the corresponding icon by button press. Selected icons were highlighted by a red frame for the duration of $1 \mathrm{~s}$, followed by a fixation cross and a variable jitter $(M=996 \mathrm{~ms}, \mathrm{SD}=418 \mathrm{~ms})$. No feedback on categorization accuracy was given. The prompted categorization during fMRI deviated from the procedure in Castelli et al. where subjects gave verbal descriptions, i.e., narratives, after each animation. The categorization used in the current study has previously been shown to be a sensitive and objective test of on-line mentalizing with the advantage of a faster assessment and more objective analysis of the participant's response [27]. Participants were familiarized to the task in a standardized training session before scanning, including three practice video clips. Task performance during the fMRI scan was evaluated in terms of overall categorization accuracy and accuracy of ToM video categorization (see Additional file 1 for additional age group-specific analyses). Analyses were performed in SPSS (IBM, version 22) using univariate analyses of covariance to assess the effects of diagnosis and age while controlling for sex, site, and IQ.
See Additional file 1 for more details on stimuli, trial structure, and task instruction.

\section{MRI data acquisition}

Data were acquired on 3-T MRI scanners, and acquisition protocols were harmonized across sites as closely as possible. Functional data was collected using an echoplanar imaging (EPI) sequence, and structural images were acquired with a high-resolution T1-weighted magnetization-prepared rapid gradient echo sequence. Data were subjected to an extensive quality assessment pipeline. Detailed information on scanning parameters and data quality control procedures is given in Additional file 1 .

\section{fMRI data analysis Preprocessing}

Functional imaging data were preprocessed using standard analysis routines implemented in SPM12 (http:// www.fil.ion.ucl.ac.uk/spm/), including slice-time correction, a two-step realignment procedure, unified segmentation and normalization to standard stereotactic space as defined by the Montreal Neurological Institute (MNI), and smoothing with an 8-mm full-width-at-halfmaximum Gaussian Kernel.

\section{Subject-level activation analysis}

Task conditions were modeled as boxcar functions that accounted for the presentation of videos and its parametric modulation. Increasing values of the parametric modulator (i.e., 1, 2, and 3) coded for increasing mentalizing demands in random, goal-directed, and ToM conditions, respectively. This approach deviates from previous ASD studies where the ToM condition was compared to the low-level baseline condition (i.e., random movement) while ignoring the goal-directed condition [7, 12, 13, 28, 29, 45, 46]. The current approach comes with the advantage that it draws on all available data, thereby maximizing statistical power and aligning with the assumption of a gradual increase of mentalizing demands across conditions [26]. We additionally modeled task effects of no interest, i.e., response selection and execution during video categorization, with boxcar functions of variable durations depending on the participant's response time. Task regressors were convolved with the canonical hemodynamic response function (HRF) and subjected as predictors to a general linear model (GLM), along with six realignment parameters to account for head motion. During first-level model estimation, data was high-pass filtered with a cutoff of $256 \mathrm{~s}$, and an autoregressive model of the first order was applied. To identify brain responses reflecting sensitivity to social significance, the effect of the parametric modulator was contrasted to the implicit baseline. 
We additionally tested for case-control differences in brain activation obtained from the original approach where animations were modeled as boxcar functions and assigned to three condition-specific regressors of interest (random, goal-directed, ToM). All remaining steps of model specification and estimation were identical. Individual contrast images were calculated for the contrast ToM > random and ToM > goal-directed, respectively.

\section{Group-level statistical inference}

Resulting contrast images were subjected to a GLM to assess the within-subject effect of task and the betweensubject effects of diagnosis and age while controlling for effects of sex and site. Age-by-diagnosis interactions were tested in an additional GLM. To assess the effect of autism traits, SRS-2 raw scores were added as an additional covariate in a separate model. Note that group was accounted for in this model, which ensures that effects were not driven by a mere difference in group means. In order to account for between-subject effects that specifically occur during development or only emerge in adulthood, two subsamples were defined: a youth sample (< 18 years) and an adult sample $(\geq 18$ years). All analyses were repeated separately in both subsamples. Across analyses, effects were evaluated at a statistical threshold of $p=0.05$, family-wise error corrected (FWE) on a voxel level across the whole brain and within the combined mask of the right pSTS and dmPFC using small volume correction (SVC; 503 voxels). Casecontrol analyses were complemented by tests for equality of distribution at selected peak voxels, using the Kolmogorov-Smirnov test with a significance level of $\alpha$ $=.05$ implemented as the ks2stat function in Matlab (version R2018b, The MathWorks, Inc.).

\section{Control analyses}

We performed control analyses to investigate whether the results were affected by various potentially influencing variables, such as demographic (acquisition site, IQ) and clinical characteristics (medication, comorbidity, current diagnostic status), task performance (categorization accuracy), functional image quality (motion and signal-to-noise ratio), and SRS informant (SRS self-report, available for adults in the ASD groups and for adolescents and adults in the TD group). We also explored whether ASD-related effects were modulated by sex (i.e., sex by diagnosis interaction). Finally, we tested whether functional brain activation related to the participants' categorization performance. See Additional file 1 for details on all control analyses.

\section{Narrative performance}

Following Castelli and colleagues [7], we assessed verbal descriptions of the animations as an additional behavioral measure. These narratives might be particularly sensitive to spontaneous mentalizing since participants are not explicitly prompted to categorize the animation. Narratives were assessed for the same animations in a separate cognitive test session that preceded the fMRI scanning on a separate day. In order to minimize participant burden, 10 animations were used $(4 \times$ ToM, $4 \times$ GD, $2 \times$ random). In short, participants were asked to spontaneously describe what was happening in each animation while watching. The responses were audio typed and analyzed based on a standardized scoring system (see Additional file 1). Here, we use the participant's mentalizing score which reflects the use of mental state terms (score of 0: absence of any mental state term, score of 1: terms that denote intentionality, e.g., "the triangle chases the other triangle," score of 2: terms that denote interactions involving theory of mind, e.g., "the small triangle tries to convince the big triangle to go out"). The mentalizing score therefore parallels the three task conditions and can thus be compared to the categorization performance during fMRI scanning.

\section{Results}

\section{Behavioral performance}

Statistical information on categorization performance during fMRI scanning is detailed in Table 2. Diagnostic groups did not differ in overall categorization accuracy or ToM video categorization accuracy. In contrast, we observed an effect of age, with better performance in older subjects. Due to the skewed distribution of values, nonparametric correlations with age are reported in Fig. 1.

Similarly to categorization performance, behavioral performance on the narratives (i.e., mean mentalizing scores for the three conditions) did not differ between cases and controls, irrespective of age $\left(F_{(1,338)} \leq 1.00, p \geq\right.$ .318). We performed a mixed multilevel analysis to test the association between fMRI categorization and mentalizing ratings along with the influence of diagnostic status and age while controlling for sex and site. Results suggest a significant association between categorization and mentalizing scores $(t=20.798, p<.001)$, with no effect of age $(t=1.632, p=.103)$ or diagnosis $(t=.71, p=$

Table 2 Video categorization accuracy for the full sample, youth sample ( $<18$ years of age), and adult sample ( $\geq 18$ years of age)

\begin{tabular}{|c|c|c|c|c|c|}
\hline Accuracy (in \%) & ASD & TD & ME diagnosis & ME age & IA age $\times$ diagnosis \\
\hline Overall & $81.4 \pm 13.4$ & $83.2 \pm 13.4$ & $F_{(1,381)}=.383, p=.536$ & $F_{(1,381)}=20.220, p<.001$ & $F_{(1,381)}=.727, p=.394$ \\
\hline ToM video & $82.7 \pm 20.5$ & $86.2 \pm 19.0$ & $F_{(1,381)}=2.181, p=.141$ & $F_{(1,381)}=28.377, p<.001$ & $F_{(1,381)}=.097, p=.755$ \\
\hline
\end{tabular}

Significant effects printed in bold; $A S D$ autism spectrum disorder, TD typical development, $M E$ main effect, IA interaction effect 


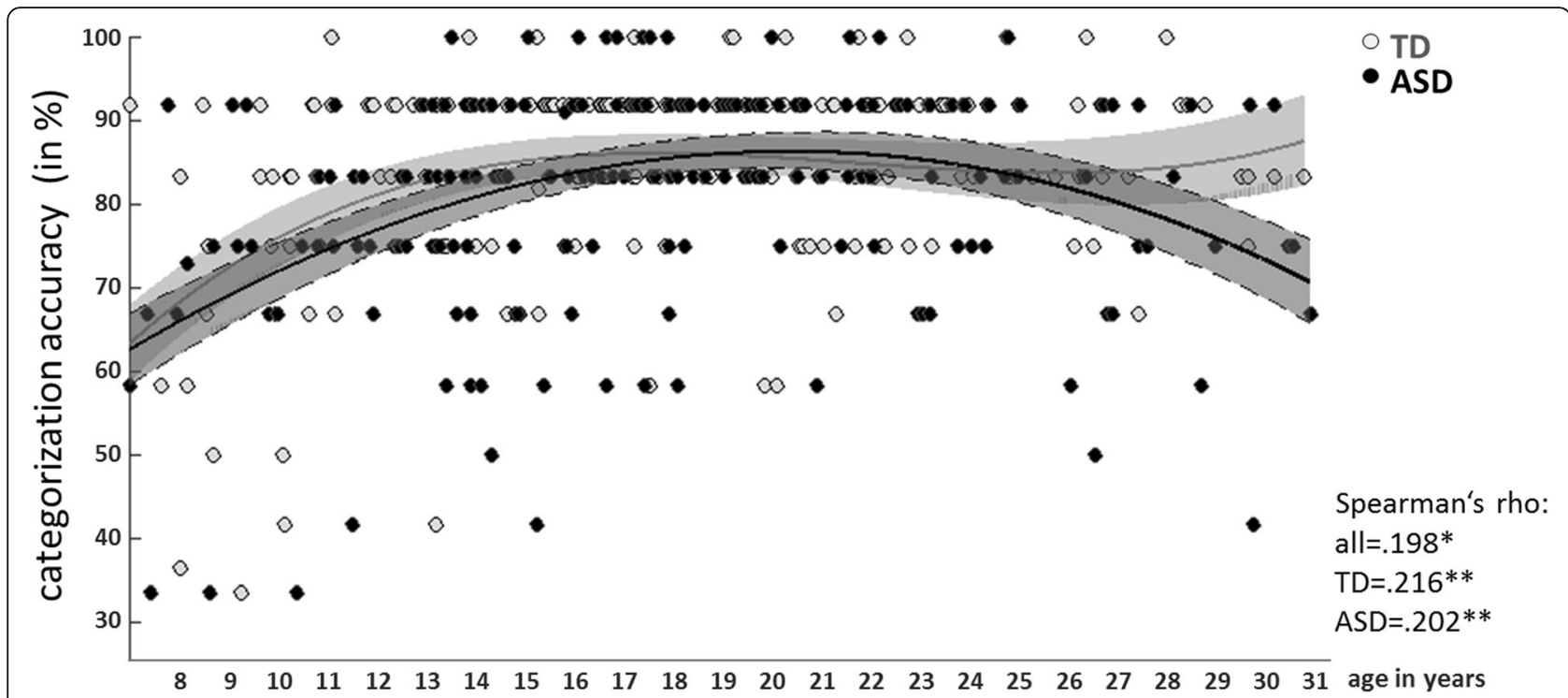

Fig. 1 Overall categorization accuracy. Linear least square regression fits (third level polynomial) for TD (gray) and ASD (black) are shown for the full sample. Shaded areas represent 95\% confidence intervals. Non-parametric correlation coefficients (Spearman's rho) are reported for all subjects and separately for TD and ASD subjects. ${ }^{*} p<.05,{ }^{* *} p<.01$

478). We additionally tested how well mean categorization accuracy corresponded to mean mentalizing scores for ToM animations. Due to the low range of possible values, non-parametric correlation analysis was used, which suggested a significant association (Kendall's tau $=.145, p=.001 ;$ Spearman's rho $=.171, p=.001)$.

\section{Brain activation}

Increasing mentalizing demands across conditions led to robust activation of key regions of the social brain, including pSTS and dmPFC, as predicted (Fig. 2a, Table 3, Additional file 1: Figure S3 and S4). We observed distinct effects of age in the full and youth samples (Fig. $2 b)$. While activation in the right anterior temporal sulcus and temporal pole decreased across the full age range (6-30 years), a specific decrease of activation was detected in the youth sample (6-18 years) in regions typically involved in the dorsal and ventral attention networks (e.g., frontal eye fields, intraparietal sulcus, anterior insula).

In the categorical analyses, brain responses to increasing mentalizing demands did not differ between diagnostic groups on the whole-brain level and within ROIs across age groups (ROI statistics for the full sample are as follows: right pSTS, main effect of diagnostic group: $F \leq 3.11, p_{\text {FWE }}(\mathrm{SVC}) \geq$ .949, diagnostic group $\times$ age interaction: $F \leq 6.35$, $p_{\text {FWE }}(\mathrm{SVC}) \geq .520$; dmPFC, main effect of diagnostic group: $F \leq 4.34, p_{\mathrm{FWE}}(\mathrm{SVC}) \geq .828$; diagnostic group $\times$ age interaction: $F \leq 7.33, p_{\mathrm{FWE}}(\mathrm{SVC})>$ .379). Cases and controls did not differ in the distribution of functional responses in selected peak voxels (see Fig. 2; all $p>.05$ ), and visual inspection of distributions did not suggest the formation of meaningful subgroups.

In contrast, the dimensional analysis uncovered an increase in dmPFC responses with increasing autism traits (full sample: peak voxel at $x=3, y=62, z=23 ; t=3.88$, $\left.p_{\text {FWE }}(\mathrm{SVC})=.011\right)$. This effect was driven by the ASD group (statistical analysis on peak voxel estimate in ASD group: $F_{(1,150)}=14.53, p<.001$; in TD group: $F_{(1,77)}=$ $.04, p=.841$; Fig. 3a). See Table 3 for a detailed list of brain regions, coordinates, and statistics.

Our additional analyses involving the ToM > random contrast did not yield any case-control difference on the whole-brain level and within regions of interest across samples (all $p_{\mathrm{FWE}}>.277$ ). The contrast involving the high-level baseline condition (ToM > goal-directed) revealed a small cluster within the dmPFC in the youth sample where individuals with ASD showed a stronger response compared with TD individuals (peak voxel at $x=9, y=$ $50, z=26 ; F=13.56, p_{\mathrm{FWE}}(\mathrm{SVC})=.034$; Fig. 3b). No other cluster emerged across search masks and samples (all $p_{\text {FWE }}>.251$ ). Dimensional analyses using these contrasts replicated the association within the dmPFC observed in the parametric modulation approach (ToM > random, full sample: peak voxel at $x=3, y=62, z=23 ; t=3.35, p_{\mathrm{FWE}}$ $(\mathrm{SVC})=.057$; youth sample: peak voxel at $x=15, y$ $\left.=50, z=35 ; t=4.20, p_{\mathrm{FWE}}(\mathrm{SVC})=.004\right)$. See Additional file 1: Table S4 for a full list of categorical and dimensional effects for the specific contrasts. 


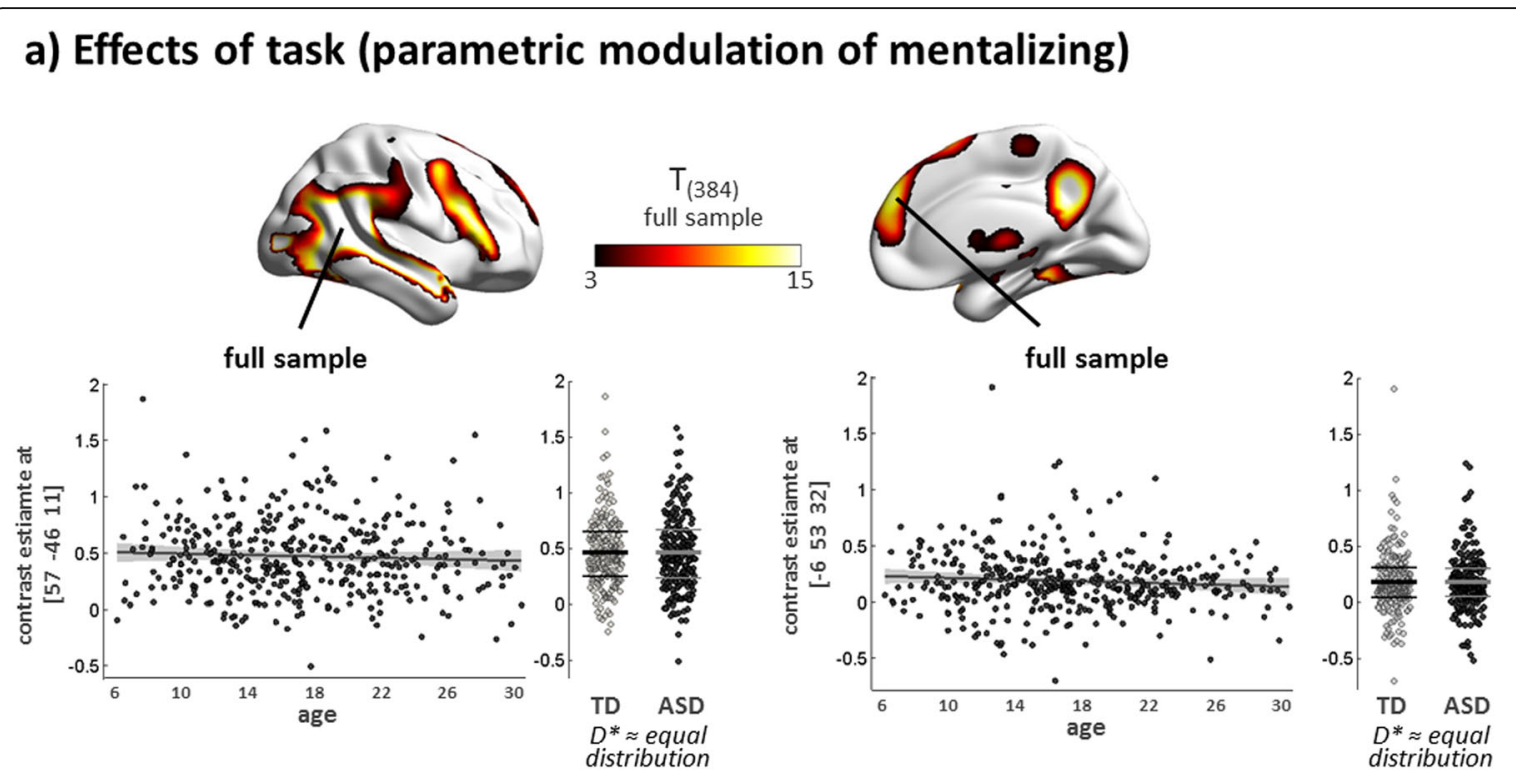

\section{b) Effects of age (parametric modulation of mentalizing)}

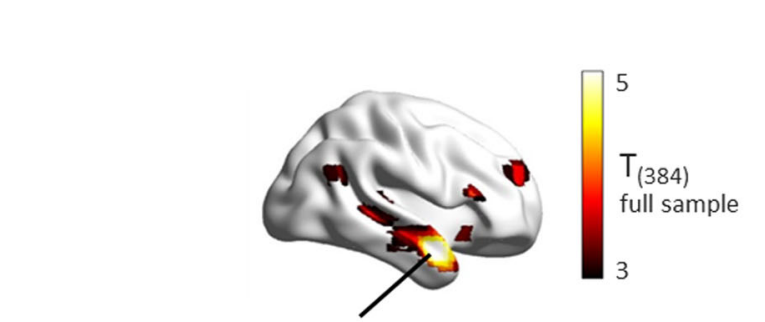

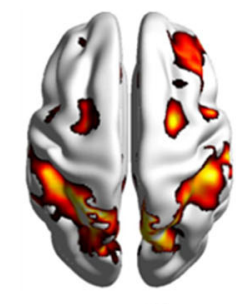

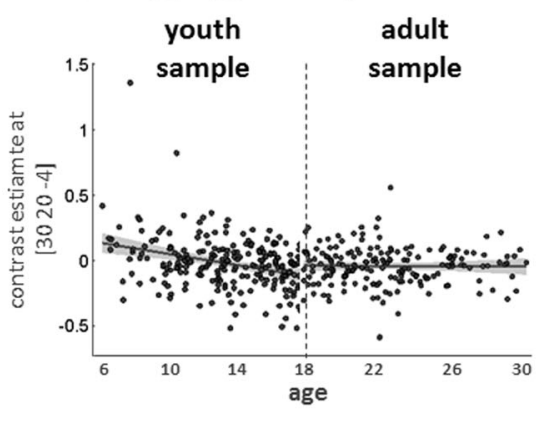

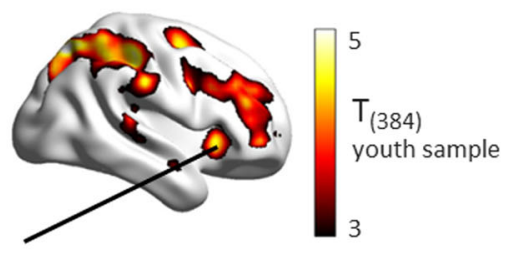

adults

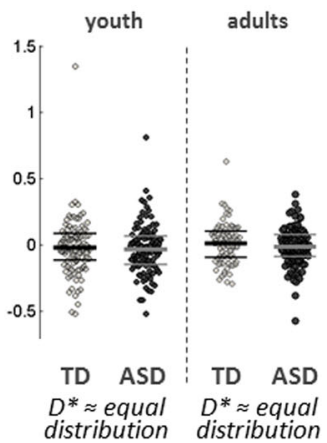

Fig. 2 Functional activation to spontaneous mentalizing elicited by increasing social significance of animated video clips. a Render brains illustrate the positive effect of task (i.e., effect of increasing social significance) in the full sample. Scatter plots highlight functional responses in selected peak voxels in the right pSTS (left) and dmPFC (right). b Render brains illustrate the positive effect of age in the full sample (left) and youth sample (right), complemented by scatter plots of peak voxels in the right anterior temporal pole (left) and right anterior insula (right). Associations with age are displayed using a linear model fit ( $95 \%$ confidence interval indicated as shaded area). Distributions of peak voxel activation in cases and controls were compared using the Kolmogorov-Smirnov test, which suggested no evidence for unequal distributions (statistics for peak at $[57,-46,11]$, full sample: $D_{(205,189)^{*}}=.065, p=.789$; for peak at $[-6,53,32]$, full sample: $D_{(205,189)^{*}}=.044, p=.990$, for peak at $[51,2,-19]$, full sample: $D_{(205,189)^{*}}=.061, p=.853$; for peak at $[30,20,-4]$, youth sample: $D_{(111,105)^{*}}=.081, p=.855$; adult sample: $D_{(93,84)^{*}}=$ $.140, p=.327)$. Distribution mean and 25th/75th percentiles are indicated as thick and thin lines within bee swarm plots, respectively. For illustration purposes, render brains are displayed at a significance threshold of $t=3$ using BrainNet Viewer (http://www.nitrc.org/projects/bnv/). $\mathrm{TD}$, typically developing; ASD, autism spectrum disorder; $D^{*}$, test statistic of the Kolmogorov-Smirnov test

\section{Control analyses}

The between-subject effects of age and diagnosis reported above were largely robust to the inclusion of additional control variables. As a notable exception, we observed an influence of site on the association between $\mathrm{dmPFC}$ and autism traits; the association dropped below the significance threshold when skipping one of the major contributing sites (KCL). The direction of the 

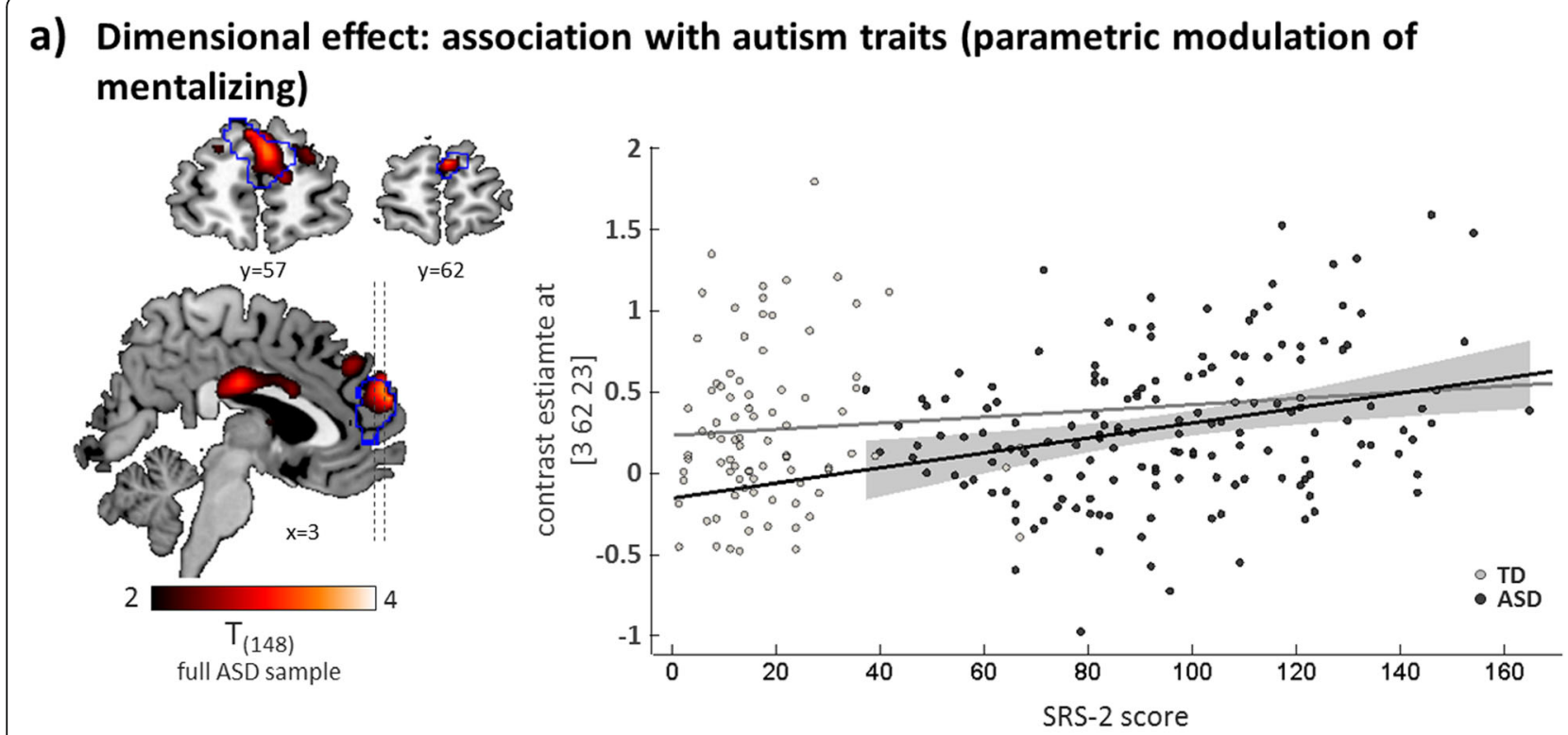

\section{b) Categorical effect: case-control difference (specific contrast: ToM > goal-directed)}

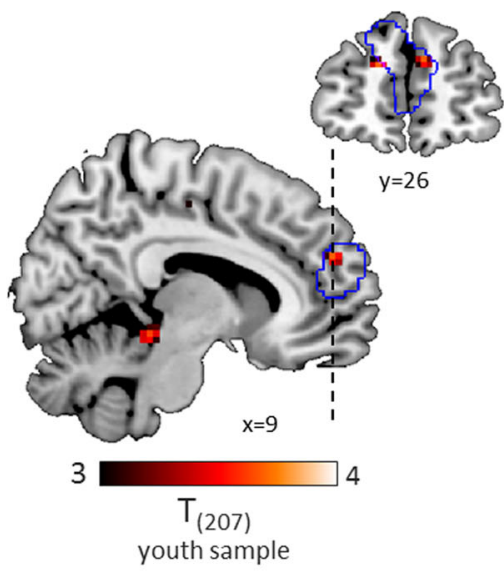

youth sample
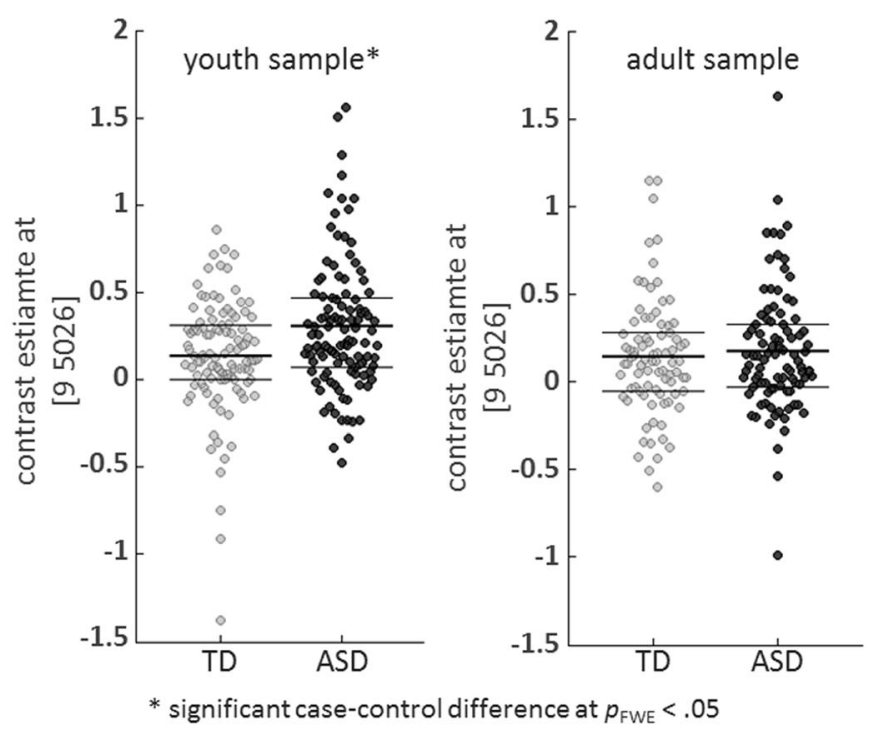

Fig. 3 a Brain sections (left) illustrate the association of a continuous measure of autism traits, as assessed as parent-reported scores of the Social Responsiveness Scale-2 (SRS-2), with functional responses to increasing mentalizing demands in the dmPFC in individuals with ASD. The outline of the ROI in the dmPFC is displayed in blue. A scatter plot (right) demonstrates the distribution of autism trait scores and peak voxel activation in cases and controls, including linear model fits for each group. The 95\% confidence interval for the significant linear model fit in the ASD group is displayed in gray. $\mathbf{b}$ Brain sections (left) illustrate the case-control difference for the specific contrast ToM > goal-directed in the $\mathrm{dmPFC}$ ROI (outlined in blue) in the youth sample. Scatter plots (right) display the distributions of peak voxel contrast estimates in the youth and adult sample, with mean and inner quartiles (25th to 75th percentile) indicated as thick and thin lines, respectively

association, however, did not change (Additional file 1: Figure S5). Follow-up analyses suggest that the KCL site effect is most plausibly explained by the fact that the KCL site contributed a (relatively) higher number of (relatively) more severely affected individuals with ASD, which boosted the association between dmPFC activation and autism trait scores. Regarding effects of medication, the exclusion of medicated individuals resulted in an attenuation of the dimensional effect in the dmPFC, which however could not be attributed to symptom severity. The direct comparison of medicated and unmedicated individuals with ASD revealed a diminished response of the insula and inferior frontal cortex in medicated children and adolescents (Additional file 1: Figure S6). No effects were observed within the canonical social brain network. We also did not observe a modulation of 
Table 3 Whole-brain parametric effects of increasing mentalizing demands on brain activation

\begin{tabular}{|c|c|c|c|c|c|c|c|c|}
\hline Effect/contrast & Sample & k & Region & $x$ & $y$ & $z$ & $t$ & $p_{\text {corr }}$ \\
\hline \multicolumn{9}{|l|}{ Within-subject effects } \\
\hline \multicolumn{9}{|l|}{ Effect of task } \\
\hline \multirow[t]{20}{*}{$\begin{array}{l}\text { Mentalizing demands: parametric } \\
\text { increase }\end{array}$} & Full & 3036 & Superior temporal gyrus & 57 & - & 11 & 28.69 & $<.001$ \\
\hline & & & Middle occipital gyrus [area hOc4lp] & 27 & $-\overline{91}$ & 2 & 24.99 & $<.001$ \\
\hline & & & Superior temporal gyrus & 48 & - & -7 & 22.04 & $<.001$ \\
\hline & & & Inferior temporal gyrus [area FG4] & 42 & - & - & 21.25 & $<.001$ \\
\hline & & & Temporal pole & 54 & 14 & $-\overline{19}$ & 16.71 & $<.001$ \\
\hline & & 4251 & Middle temporal gyrus & $\begin{array}{l}- \\
54\end{array}$ & $\begin{array}{l}- \\
52\end{array}$ & 14 & 26.84 & $<.001$ \\
\hline & & & Middle occipital gyrus [area hOc4lp] & - & - & -4 & 23.03 & $<.001$ \\
\hline & & & Fusiform gyrus [area FG4] & - & - & - & 21.55 & $<.001$ \\
\hline & & & Cerebellum [lobule Vlla crusl (hem)] & - & - & - & 16.49 & $<.001$ \\
\hline & & & Inferior frontal gyrus, pars triangularis & $\begin{array}{l}- \\
51\end{array}$ & 20 & 20 & 16.40 & $<.001$ \\
\hline & & 1115 & Precuneus & 6 & - & 38 & 18.85 & $<.001$ \\
\hline & & 868 & $\begin{array}{l}\text { Inferior frontal gyrus, pars triangularis [area } \\
45 \text { ] }\end{array}$ & 51 & 26 & 17 & 14.96 & $<.001$ \\
\hline & & & Precentral gyrus & 45 & 5 & 50 & 12.57 & $<.001$ \\
\hline & & 925 & Dorsomedial prefrontal gyrus & -6 & 53 & 32 & 13.98 & $<.001$ \\
\hline & & & Supplementary motor area & 9 & 17 & 65 & 10.82 & $<.001$ \\
\hline & & 56 & Cerebellum [lobule IX (hem)] & -6 & - & - & 11.02 & $<.001$ \\
\hline & & 553 & Thalamus [thalamus: prefrontal] & 9 & - & 8 & 9.46 & $<.001$ \\
\hline & & 27 & Cerebellum vermis [lobule I IV (hem)] & 0 & - & - & 6.06 & $<.001$ \\
\hline & & 6 & Middle cingulate cortex & 0 & - & 41 & 5.44 & .001 \\
\hline & & 2 & Middle frontal gyrus & $-\overline{24}$ & 23 & 44 & 4.60 & .025 \\
\hline
\end{tabular}

Between-subject effects

Effect of age

Age: linear decrease

Full

12 Medial temporal pole

Age: linear decrease

Youth

249 Superior parietal lobule [area 7P (SPL)]

19

$\begin{array}{lllll}- & - & 56 & 6.37 & .000 \\ 27 & 58 & & & \end{array}$

207 Superior parietal lobule [area 7P (SPL)]

$\begin{array}{lllll}18 & - & 53 & 5.75 & .000\end{array}$

Precuneus

$\begin{array}{lllll}-6 & - & 44 & 5.45 & .001\end{array}$

Superior parietal lobule [area 7A (SPL)]

$\begin{array}{lllll}18 & - & 62 & 5.30 & .002\end{array}$

35 Inferior parietal lobule [area hIP1 (IPS)]

$\begin{array}{llll}-\quad & - & 38 & 5.14 \quad .004\end{array}$ 
Table 3 Whole-brain parametric effects of increasing mentalizing demands on brain activation (Continued)

\begin{tabular}{|c|c|c|c|c|c|c|c|c|}
\hline Effect/contrast & Sample & k & Region & $x$ & $y$ & $z$ & $t$ & $p_{\text {corr }}$ \\
\hline & & & & 36 & 40 & & & \\
\hline & & & Inferior parietal lobule [area PFt (IPL)] & $\begin{array}{l}- \\
48\end{array}$ & $-\overline{34}$ & 41 & 4.78 & .017 \\
\hline & & 35 & Inferior parietal lobule [area 2] & 48 & $\begin{array}{l}- \\
34\end{array}$ & 47 & 5.01 & .007 \\
\hline & & 13 & Middle cingulate gyrus & 6 & 26 & 38 & 4.85 & .013 \\
\hline & & & Anterior cingulate gyrus & 6 & 32 & 26 & 4.60 & .034 \\
\hline & & 20 & Superior frontal gyrus & 24 & -1 & 47 & 4.83 & .014 \\
\hline & & 11 & Anterior insula & 30 & 20 & -4 & 4.77 & .017 \\
\hline & & 5 & Supramarginal gyrus (area PFt (IPL)] & 63 & $\frac{-}{22}$ & 35 & 4.72 & .021 \\
\hline & & 8 & Inferior parietal lobule [area 7PC (SPL)] & 33 & $\begin{array}{l}- \\
46\end{array}$ & 53 & 4.72 & .021 \\
\hline & & 2 & Middle frontal gyrus & 45 & 38 & 20 & 4.60 & .034 \\
\hline & & 1 & Postcentral gyrus [area 4p] & 33 & $\begin{array}{l}- \\
31\end{array}$ & 50 & 4.57 & .037 \\
\hline
\end{tabular}

Effect of diagnosis (categorical)

No sign. effect

Effect of parent-reported autism traits (dimensional)

Linear increase

Linear increase

$$
\begin{aligned}
& \text { Full (no TD } \\
& \text { adult }{ }^{1} \text { ) }
\end{aligned}
$$

Youth
4
Inferior parietal lobule [area PFm (IPL)]
10svc dmPFC (combined mask)
$15_{\text {sve }}$ dmPFC (combined mask)
23sve dmPFC (combined mask)
8svc dmPFC (combined mask)

$$
\text { (1) }
$$$$
\begin{array}{lllll}
54 & - & 38 & 4.82 & .021 \\
& 55 & & & \\
3 & 62 & 23 & 3.88 & .011_{\text {SVC }} \\
-9 & 53 & 38 & 3.49 & .035_{\text {SVC }} \\
3 & 56 & 26 & 3.77 & .016_{\text {SVC }} \\
- & 50 & 35 & 3.43 & .046_{\text {SVC }} \\
15 & & & &
\end{array}
$$

Regions were classified according to the Automated Anatomical Labeling Atlas [47]. If applicable, functional labels were added in square brackets based on Anatomical Probability Maps (Anatomy toolbox [48]). $x-, y$-, and $z$-coordinates (MNI) and statistical information refer to peak voxels in the identified clusters. $p$ values are adjusted for family-wise error correction for multiple comparisons across the whole brain, or across the combined mask of the right $\mathrm{pSTS}$ and dmPFC using small volume correction (SVC). Age and sex were included as covariates in the analysis. SPL superior parietal lobule, IPS intraparietal sulcus, IPL inferior parietal lobule, TD typically developing, $p S T S$ posterior superior temporal sulcus, dmPFC dorsomedial prefrontal cortex

${ }^{1}$ no parent-reported SRS-scores available for TD adults

ASD-related effects by sex. Likewise, follow-up analyses do not suggest an impact of categorization accuracy on the association between $\mathrm{dmPFC}$ and autism traits. See Additional file 1 for detailed information on the results of all control analyses.

\section{Discussion}

In this to date largest study on the neurofunctional development of the social brain, we characterized social brain activation during mentalizing in a deeply phenotyped sample of individuals with ASD and TD controls. Besides showing a robust effect of task, we demonstrate (1) that functional responses of core regions of the social brain are well developed by the age of 6 while age-related changes occur in a distributed set of brain regions typically implicated in attention and executive control, (2) that categorical case-control comparisons between TD and ASD participants do not reveal clear-cut group differences in mean and distribution of functional activation measures, and (3) that a dimensional analysis approach might offer higher sensitivity to detect ASD-related effects.

\section{Robust effects of social animations on functional activation}

Across both groups, we replicated the effect of increased activation in key regions of the social brain $[7,10]$ that scaled with the mentalizing demands imposed by the animations. Resulting activation patterns are highly similar to those obtained from the comparison of the ToM condition to the low-level baseline condition, which suggests that effects are mainly driven by the ToM condition. Activation was strongest in the bilateral pSTS and adjacent temporal and occipital cortices, reflecting the central role of sensory bottom-up processing in this task where social meaning is extracted from the spatial constellation of the moving shapes. We also observed robust 
responses of the dmPFC, IFG, precuneus, and temporal poles, as previously described $[10,29]$.

\section{Effects of age}

Across both groups, younger participants were less accurate in video categorization. However, this age effect in behavior was not reflected on the level of the social brain circuitry where functional activation was largely unaffected by age. This is in contrast to several studies on mentalizing that reported higher social brain activation in younger subjects in various regions, such as medial frontal, inferior frontal, or temporal areas (e.g., [46, 49-51]). Common interpretations are less efficient processing or different cognitive strategies [52] that might parallel ongoing structural maturation [53]. In our large sample, however, these effects did not replicate, neither across the full sample nor when splitting the sample into two subsamples to approximate non-linear age effects, and despite the fact that we had a $95 \%$ power to detect small-to-moderate effects. An exception is the right temporal pole that showed a gradual decrease of activation from childhood to adulthood, potentially reflecting its protracted structural development [53]. We propose two explanations for the lack of age effects on social brain activation. First, while previous reports were fairly consistent in reporting stronger responses in younger individuals, they were less consistent in the localization of these effects. For instance, medial prefrontal effects ranged from subgenual to dorsal areas. This heterogeneity likely results from methodological differences and limited statistical power, along with selection and reporting biases (e.g., [25, 44, 54-57]), which questions the generalizability of previous findings [25]. Second, spontaneous mentalizing is a fundamentally basic skill akin to tracking beliefs, an ability already seen in toddlers ([58, 59], but see [60] for a critical discussion). This suggests an early development of its neurofunctional basis. In fact, a recent study demonstrated the social brain network to be functionally distinct and robustly recruited in 3-year-old children, independent from explicit mentalizing skills [61]. Similarly, a recent study in 50 children and adolescents did not find an effect of age on several measures of functional connectivity of the social brain [62]. Overall, these and our findings suggest that across study populations, the core neurofunctional network for mentalizing is largely set by the age of 6 and does not undergo gross changes from mid-childhood to adulthood. This does not preclude the existence of specific developmental alterations in conditions like ASD, as discussed below.

Outside the social brain, however, we did observe an effect of age. A distributed set of brain regions including the frontal eye fields, intraparietal sulcus, and anterior insula demonstrated a gradual decrease of activation from childhood to early adulthood. These regions have been identified as key hubs of the dorsal and ventral attention systems that support top-down controlled orienting of attention and bottom-up-mediated reallocation of attention to salient events [63]. Age effects were also evident in dorsolateral prefrontal areas that have been suggested to support the flexible switch between both attention systems [63]. According to our data, children and young adolescents might recruit attentional resources more strongly than older adolescents and adults when inferring social meaning from the animated shapes. Along with our observation of better task performance in adults, these findings suggest an important contribution of domain-general networks to social cognition [64].

\section{Effects of diagnosis}

Contrary to our hypothesis, we did not observe robust case-control differences in both behavioral measures of mentalizing (i.e., categorization and narratives) and in social brain responses to increasing levels of mental state attribution. We also did not see a case-control difference when comparing the ToM condition to the low-level baseline condition. However, when comparing the ToM condition to the goal-directed condition, one cluster emerged within our dmPFC key region, showing a higher average response in ASD, which was restricted to the youth sample. Across these analyses, we did not see major differences in the distributions of functional responses between cases and controls or any indication for the formation of subgroups within the ASD sample. Beyond the categorical approach, we observed a moderate and positive association between a continuous measure of autistic traits and mentalizing-related activation in the dmPFC. This association was only observed in the ASD group and predicted higher brain responses in individuals with higher autistic traits, irrespective of age.

The lack of robust case-control differences needs to be discussed in the light of the positive findings by Castelli et al. [7] and others using the animated shapes task [12-14]. First, as discussed above in the context of non-replicable age effects, one possible explanation is that previously reported case-control differences in studies with low statistical power reflect idiosyncratic characteristics of the specific sample and experimental procedure. A recent study using simulated and empirical fMRI data provided compelling evidence that between-subject effects, such as casecontrol differences and brain-behavior relationships, are usually weak and spatially distributed [44]. It is plausible that ASD-related effects generally also follow this weak and diffuse pattern. As a result, commonly used sample sizes in earlier ASD studies $(n=10-30)$, which were sufficiently powered to detect strong and 
localized within-subject effects (e.g., effect of an experimental condition), might have lacked the statistical power to detect robust effects related to ASD. A consequence of this power failure is an overestimation of effect sizes, including spurious effects due to random error, which produced a heterogeneous pattern of putative ASD-related effects that do not replicate in follow-up studies. Second, the continuous revision of diagnostic criteria and the fact that the autism diagnosis is not stationary over time might further impact the comparability to older studies [65]. A third explanation is that earlier findings obtained in smaller, more homogeneous, and potentially more severely affected samples might relate to specific subsamples, or "strata", within the ASD population. The LEAP cohort was purposely sampled to enable indepth experimental characterization of potential biomarkers (including MRI scans) and therefore excluded individuals with low IQ $(<50)$ and most likely individuals with the most severe autistic presentations. However, the sample does allow us to investigate the heterogeneity of the ASD population with respect to age, sex, comorbidities, and symptom profiles. In the current study, we have not leveraged this heterogeneity for stratification purposes yet, but tested for differences in average response while controlling for these sources of variance, which might have resulted in reduced sensitivity for subsample-specific effects. For instance, despite covering the full range of symptom severity, the LEAP cohort is, on average, rather mildly affected, which might have contributed to the negative findings in case-control comparisons. However, our control analysis restricted to more severely affected individuals (see section 6.8 in the Additional file 1) did not change the results, which is not consistent with this potential explanation for our negative findings, at least in our sample. Fourth, despite extensive standard operation procedures, the multi-site acquisition design is a potential source of unwanted variance which might have similarly resulted in reduced sensitivity for smaller-sized effects [66]. We addressed this variance in our analyses which did not suggest a systematic confounding effect of site. Fifth, in contrast to Castelli et al. [7], participants were prompted to focus on the category of the animation, which introduces an explicit component to this otherwise implicit, or "spontaneous," mentalizing task. It has been suggested that spontaneous mentalizing is the key difference between autism and typical development and that individuals with ASD can explicitly mentalize when prompted to do so [2]. The explicit categorization might therefore have reduced, or even abolished, mentalizing-related case-control differences in our study [67]. While this interpretation needs to be tested in future large-scale studies, it is challenged by earlier reports of behavioral case-control differences using the prompted categorization approach $[13,27]$. In addition, our analyses suggest a significant association between prompted categorization and a measure of spontaneous mentalizing in the same individuals. Finally, the intriguingly simplistic yet powerful effect of the animated shapes is hypothesized to originate from an efficient, early developing mentalizing system that offers little room for manipulation by explicit strategies $[9,58]$, which is supported by neurofunctional evidence in TD participants [45].

Our categorical approach of comparing cases and controls therefore does not lend support to the hypothesis that social brain function during mentalizing elicited by animated shapes is a one-to-one correlate of social impairments in individuals with ASD. That said, however, both categorical and dimensional analyses converged on two small-to-moderate effects in our dmPFC key region. The mentalizing-specific activation in the dmPFC was stronger in younger individuals with ASD compared to TD individuals. Similarly, dmPFC activation increased with the degree to which an individual with ASD demonstrates autistic behavior as assessed by parentreported autistic traits. While counterintuitive at first sight, a possible explanation is a greater need for a compensatory recruitment of the $\mathrm{dmPFC}$ in younger individuals and with increasing autism traits, an effect that was recently introduced as "camouflaging" in the context of mentalizing [68]. This interpretation is also in line with the absence of a dimensional effect in TD individuals, although this seems to be primarily due to the limited variance in autism trait scores. Supplemental analyses do not suggest an association of dmPFC responses with categorization accuracy, which can similarly be reconciled with the hypothesis of successful camouflaging. We acknowledge, however, that caution is warranted when interpreting our dimensional finding since our control analyses point to a potential effect of site and since this association occurs within the neurotypical range, as suggested by the lack of an overall case-control difference. Further studies are needed to follow up on this finding and its potential biological meaning.

What does our observation of largely overlapping social brain responses in individuals with ASD and TD individuals add to current theories of autism? The animated shapes task is hypothesized to mainly draw upon implicit mentalizing which is required for fastpaced real-life interactions $[9,58]$ and which is suggested to be specifically impaired in autistic individuals [2]. With the caveat of an explicit component introduced by the prompted categorization, our results provide no evidence of an altered recruitment of its neural correlates in autism, at least in terms of a consistent alteration 
in the strength of activation. This leaves open the possibility of idiosyncratic, non-converging alterations in brain activation which is not captured by conventional fMRI analyses based on group means [65, 69]. Likewise, the dysconnectivity account of autism postulates alterations in functional connectivity to underlie behavioral and clinical impairments [70]. Measures of functional connectivity and their modulation by mentalizing demands might therefore be more promising for biomarker research. Additional, likely multiple, causes might contribute to the exacerbation of socio-communicative impairments of autistic individuals in everyday life. These may include cascading effects of impairments in other domains (e.g., sensory abnormalities [71]), a lower propensity to adopt the intentional stance [72], reduced motivational salience of social and non-social stimuli [73], or direct or indirect effects of comorbid conditions (e.g., alexithymia [74]).

\section{Limitations}

Despite an extensive set of control analyses, we cannot rule out additional sources of variance that remain unaddressed or poorly addressed, such as specific effects of medication, and might thereby prevent the detection of effects of interest despite our well-powered sample. Regarding sample characteristics, we acknowledge that the proportion of more severely affected individuals was comparatively low. We also did not include individuals with low IQ (< $50)$, which is a common limitation in imaging studies where experimental demands are too burdening for low-functioning individuals. This restriction compromises the heterogeneity of the LEAP cohort and its representativeness for the ASD population. Regarding our experimental protocol, this task uses graded levels of mental state attribution as a correlate for ToM, which might be less sensitive to capture specific effects of mentalizing on the neural and behavioral level. Small methodological differences to the original study by Castelli et al. [7] might have contributed to the lack of case-control differences on the behavioral level (e.g., use of 3-point instead of 6point scale for obtaining narrative scores). Overall, the pattern of strong within-subject effects and small-to-absent between-subject effects might partly result from substantial between-subject heterogeneity, which may be exacerbated in multicenter designs, but is a feature of samples that strive for representativeness for the underlying population. While this is the case for the current study, we followed recommended procedures to ensure optimal alignment of data acquisition between centers [66] and aimed for a deep multimodal characterization of our study participants [24, 37]. Furthermore, our distributional observations do not support the interpretation of distinct subgroups of participants with ASD masking group mean effects.

\section{Conclusions}

In the current report, we examined social brain correlates of mentalizing in ASD in a large and well-powered task-based fMRI dataset. Developmental effects were observed in younger individuals who showed a stronger modulation of attention-related brain areas by mentalizing demands. Against our expectation, we did not replicate previous observations of reduced activation in individuals with ASD. Instead, brain responses in autistic individuals were robustly typical in that they showed the expected effects of task in the social brain circuitry. We therefore conclude that time-locked functional activation in an animated shapes task does not inform the neurobiological basis of the mind-blindness account of ASD. This calls for the interrogation of different neural phenotypes, in particular connectivity and connectomic measures such as those derived from graph theory, which may access brain functional interaction not captured by the activation measures investigated here. Furthermore, our work clearly demonstrates the importance of novel research strategies that go beyond case-control comparisons but rather target the heterogeneity in ASD itself (e.g., [65]), an opportunity that is offered by largescale data sets such as LEAP.

\section{Supplementary information}

Supplementary information accompanies this paper at https://doi.org/10. 1186/s13229-020-0317-x.

\section{Additional file 1. Supplementary Material.}

\section{Acknowledgements}

We thank all participants and their families for their efforts to participate in the study.

Group authorship: Jumana Ahmad, Sara Ambrosino, Bonnie Auyeung, Tobias Banaschewski, Simon Baron-Cohen, Sarah Baumeister, Christian F. Beckmann, Sven Bölte, Thomas Bourgeron, Carsten Bours, Michael Brammer, Daniel Brandeis, Claudia Brogna, Yvette de Bruijn, Jan K. Buitelaar, Bhismadev Chakrabarti, Tony Charman, Ineke Cornelissen, Daisy Crawley, Flavio Dell'Acqua, Guillaume Dumas, Sarah Durston, Christine Ecker, Jessica Faulkner, Vincent Frouin, Pilar Garcés, David Goyard, Lindsay Ham, Hannah Hayward, Joerg Hipp, Rosemary Holt, Mark H. Johnson, Emily J.H. Jones, Prantik Kundu, Meng-Chuan Lai, Xavier Liogier D’ardhuy, Michael V. Lombardo, Eva Loth, David J. Lythgoe, René Mandl, Andre Marquand, Luke Mason, Maarten Mennes, Andreas MeyerLindenberg, Carolin Moessnang, Nico Mueller, Declan G.M. Murphy, Bethany Oakley, Laurence O’Dwyer, Marianne Oldehinkel, Bob Oranje, Gahan Pandina, Antonio M. Persico, Barbara Ruggeri, Amber Ruigrok, Jessica Sabet, Roberto Sacco, Antonia San José Cáceres, Emily Simonoff, Will Spooren, Julian Tillmann, Roberto Toro, Heike Tost, Jack Waldman, Steve C.R. Williams, Caroline Wooldridge, and Marcel P. Zwiers.

\section{Authors' contributions}

AML, JB, DM, EL, SBC, SB, SD, TP, WS, TC, TB, DB, and HT designed the study. JT, SB, MO, SA, CB, DC, HH, RH, ASJC, and the EU-AIMS LEAP group collected the data. CM and SB conducted the analyses. DG, CB, FDA, CE, VF, MJ, EJ, $M C L, M V L$, and LM made substantial contributions to the interpretation of 
the data. CM and AML wrote the first and final draft of the manuscript. All author(s) read and approved the final manuscript.

\section{Funding}

This project has received funding from the Innovative Medicines Initiative 2 Joint Undertaking under grant agreement No 777394 for the project AIMS-2TRIALS. This Joint Undertaking receives support from the European Union's Horizon 2020 research and innovation programme and EFPIA and AUTISM SPEAKS, Autistica, SFARI. Additional funding was received by the European Community's Seventh Framework Programme under the grant agreement no. 602805 (Project EU-AGGRESSOTYPE) and no. 602450 (Project EUIMAGEMEND), and by the German Federal Ministry of Education and Research under the grant no. 01ZX1314GM (Project IntegraMent) and grant no. 01GQ1102. C.M. is a recipient of an Olympia-Morata grant of the University Heidelberg.

\section{Availability of data and materials}

The datasets generated and/or analyzed during the current study are not publicly available due to an embargo period but are available from the corresponding author on reasonable request.

\section{Ethics approval and consent to participate}

The study was approved by the local ethical committees of the participating centers, and written informed consent was obtained from all participants or their legal guardians (for participants $<18$ years).

\begin{tabular}{lll}
\hline Site & Ethics committee & $\begin{array}{l}\text { ID/reference } \\
\text { no. }\end{array}$ \\
\hline KCL, & London Queen Square Health Research, & $13 /$ LO/1156 \\
UCAM & Authority Research Ethics Committee & \\
RUNMC, & Instituut Waarborging Kwaliteit en Veiligheid, & $2013 / 455$ \\
UMCU & Commissie Mensgebonden Onderzoek, Regio & \\
& Arnhem-Nijmegen (Radboud University Medical & \\
& Centre Institute Ensuring, Quality and Safety & \\
& Committee on Research Involving Human Sub- & \\
& jects Arnhem-Nijmegen) & 2014-540 N- \\
CIMH & UMM Universitatsmedizin Mannheim, & MA \\
& Medizinische Ethik Commission II (UMM & \\
& University Medical Mannheim, Medical Ethics & \\
& Commission II) & \\
UCBM & Università Campus Bio-Medico di Roma, Comi- & $18 / 14$ PAR \\
& tato Etico (University Campus Bio-Medical Ethics & ComET CBM \\
& Committee of Rome) & \\
\hline
\end{tabular}

\section{Consent for publication}

Not applicable

\section{Competing interests}

$A M-L$ has received consultant fees from American Association for the Advancement of Science, Atheneum Partners, Blueprint Partnership, Boehringer Ingelheim, Daimler und Benz Stiftung, Elsevier, F. Hoffmann-La Roche, ICARE Schizophrenia, K. G. Jebsen Foundation, L.E.K Consulting, Lundbeck International Foundation (LINF), R. Adamczak, Roche Pharma, Science Foundation, Sumitomo Dainippon Pharma, Synapsis Foundation-Alzheimer Research Switzerland, and System Analytics, and has received lectures fees including travel fees from Boehringer Ingelheim, Fama Public Relations, Institut d'investigacions Biomèdiques August Pi i Sunyer (IDIBAPS), Janssen-Cilag, Klinikum Christophsbad, Göppingen, Lilly Deutschland, Luzerner Psychiatrie, LVR Klinikum Düsseldorf, LWL Psychiatrie Verbund Westfalen-Lippe, Otsuka Pharmaceuticals, Reunions i Ciencia S. L., Spanish Society of Psychiatry, Südwestrundfunk Fernsehen, Stern TV, and Vitos Klinikum Kurhessen. WM has received lecture or travel fees from Pfizer, Grünenthal, University of Zürich, International Association for the Study on Pain (IASP), and European Federation of IASP Chapters (EFIC). SB discloses that he has in the last 5 years acted as an author, consultant or lecturer for Shire, Medice, Roche, Eli Lilly, Prima Psychiatry, GLGroup, System Analytic, Ability Partner, Kompetento, Expo Medica, Clarion Healthcare, and Prophase. He receives royalties for textbooks and diagnostic tools from Huber/Hogrefe, Kohlhammer, and UTB. The other authors declare that they have no competing interests.

\section{Author details}

${ }^{1}$ Department of Psychiatry and Psychotherapy, Central Institute of Mental Health, Medical Faculty Mannheim / University of Heidelberg, Mannheim, Germany. ${ }^{2}$ Department of Child and Adolescent Psychiatry and Psychotherapy, Central Institute of Mental Health, Medical Faculty Mannheim / University of Heidelberg, Mannheim, Germany. ${ }^{3}$ Department of Psychology, Institute of Psychiatry, Psychology \& Neuroscience, King's College London, London, UK. ${ }^{4}$ Department of Applied Psychology: Health, Development, Enhancement, and Intervention, University of Vienna, Vienna, Australia. ${ }^{5}$ Neurospin Centre CEA, Saclay, Gif sur Yvette, France. ${ }^{6}$ Department of Psychiatry, UMC Utrecht Brain Center, Utrecht, The Netherlands. ${ }^{7}$ Autism Research Centre, Department of Psychiatry, University of Cambridge, Cambridge, UK. ${ }^{8}$ Donders Institute for Brain, Cognition and Behavior, Radboud University, Nijmegen, The Netherlands. ${ }^{9}$ Department of Cognitive Neuroscience, Radboud University Medical Center, Nijmegen, The Netherlands. ${ }^{10}$ Center of Neurodevelopmental Disorders (KIND), Centre for Psychiatry Research, Department of Women's and Children's Health, Karolinska Institutet and Child and Adolescent Psychiatry, Stockholm Health Care Services, Stockholm County Council, Stockholm, Sweden. ${ }^{11}$ Curtin Autism Research Group, School of Occupational Therapy, Social Work and Speech Pathology, Curtin University, Perth, Western Australia, Australia. ${ }^{12}$ Department of Forensic and Neurodevelopmental Sciences, Institute of Psychiatry, Psychology \& Neuroscience, King's College London, London, UK. ${ }^{13}$ Sackler Institute for Translational Neurodevelopment, Institute of Psychiatry, Psychology \& Neuroscience, King's College London, London, UK.

${ }^{14}$ Department of Child and Adolescent Psychiatry, Psychosomatics and Psychotherapy, University Hospital Frankfurt am Main, Goethe University, Frankfurt, Germany. ${ }^{15}$ Centre for Brain and Cognitive Development, Birkbeck, University of London, London, UK. ${ }^{16}$ Centre for Addiction and Mental Health and The Hospital for Sick Children, Department of Psychiatry, University of Toronto, Toronto, Canada. ${ }^{17}$ Department of Psychiatry, National Taiwan University Hospital and College of Medicine, Taipei, Taiwan. ${ }^{18}$ Department of Psychology, University of Cyprus, Nicosia, Cyprus. ${ }^{19} \mathrm{Child}$ and Adolescent Neuropsychiatry Unit, "Gaetano Martino" University Hospital, University of Messina, Messina, Italy. ${ }^{20}$ Mafalda Luce Center for Pervasive Developmental Disorders, University Campus Bio-Medico, Milan, Italy. ${ }^{21}$ Roche

Pharmaceutical Research and Early Development, NORD Discovery and Translational Area, Roche Innovation Center Basel, Basel, Switzerland.

${ }^{22}$ Karakter Child and Adolescent Psychiatry University Centre, Nijmegen, The Netherlands. ${ }^{23}$ Neuroscience Center Zurich, University of Zurich and ETH Zurich, Zurich, Switzerland. ${ }^{24}$ Center for Integrative Human Physiology Zurich, University of Zurich, Zurich, Switzerland.

Received: 2 April 2019 Accepted: 23 January 2020

Published online: 22 February 2020

\section{References}

1. Baron-Cohen S. Mindblindness: an essay on autism and theory of mind. Boston: MIT Press/Bradford Books; 1995.

2. Frith U. Emanuel Miller lecture: confusions and controversies about Asperger syndrome. Journal of child psychology and psychiatry, and allied disciplines. 2004:45(4):672-86.

3. Senju A, Southgate $V$, White $S$, Frith U. Mindblind eyes: an absence of spontaneous theory of mind in Asperger syndrome. Science. 2009; 325(5942):883-5.

4. Bolte S, Ciaramidaro A, Schlitt S, Hainz D, Kliemann D, Beyer A, et al. Training-induced plasticity of the social brain in autism spectrum disorder, Br J Psychiatry. 2015;207(2):149-57.

5. Scheeren AM, de Rosnay M, Koot HM, Begeer S. Rethinking theory of mind in high-functioning autism spectrum disorder. J Child Psychol Psychiatry. 2013:54(6):628-35.

6. Peterson CC, Garnett M, Kelly A, Attwood T. Everyday social and conversation applications of theory-of-mind understanding by children with autism-spectrum disorders or typical development. Eur Child Adolesc Psychiatry. 2009;18(2):105-15.

7. Castelli F, Frith C, Happe F, Frith U. Autism, Asperger syndrome and brain mechanisms for the attribution of mental states to animated shapes. Brain. 2002;125(Pt 8):1839-49. 
8. Heider $H$, Simmel M. An experimental study of apparent behavior. Am J Psychol. 1944;57(2):243-59.

9. Mar RA, Macrae CN. Triggering the intentional stance. Novartis Foundation Symp. 2007;278:111-20 discussion 20-33, 216-21.

10. Schurz M, Radua J, Aichhorn M, Richlan F, Perner J. Fractionating theory of mind: a meta-analysis of functional brain imaging studies. Neurosci Biobehav Rev. 2014;42:9-34.

11. Loth E, Spooren W, Ham LM, Isaac MB, Auriche-Benichou C, Banaschewski T, et al. Identification and validation of biomarkers for autism spectrum disorders. Nat Rev Drug Discov. 2015;15(1):70-3.

12. Kana RK, Keller TA, Cherkassky VL, Minshew NJ, Just MA. Atypical frontalposterior synchronization of Theory of Mind regions in autism during mental state attribution. Social neuroscience. 2009;4(2):135-52.

13. Kana RK, Maximo JO, Williams DL, Keller TA, Schipul SE, Cherkassky VL, et al. Aberrant functioning of the theory-of-mind network in children and adolescents with autism. Mol Autism. 2015;6:59.

14. Weisberg J, Milleville SC, Kenworthy L, Wallace GL, Gotts SJ, Beauchamp MS, et al. Social perception in autism spectrum disorders: impaired category selectivity for dynamic but not static images in ventral temporal cortex. Cerebral Cortex. 2014;24(1):37-48.

15. Patriquin MA, DeRamus T, Libero LE, Laird A, Kana RK. Neuroanatomical and neurofunctional markers of social cognition in autism spectrum disorder. Hum Brain Mapping. 2016;37(11):3957-78.

16. Philip RC, Dauvermann MR, Whalley HC, Baynham K, Lawrie SM, Stanfield AC. A systematic review and meta-analysis of the fMRI investigation of autism spectrum disorders. Neurosci Biobehav Rev. 2012;36(2):901-42.

17. Dickstein DP, Pescosolido MF, Reidy BL, Galvan T, Kim KL, Seymour KE, et al. Developmental meta-analysis of the functional neural correlates of autism spectrum disorders. J Am Acad Child Adolesc Psychiatry. 2013;52(3):279-89 e16.

18. Sugranyes G, Kyriakopoulos M, Corrigall R, Taylor E, Frangou S. Autism spectrum disorders and schizophrenia: meta-analysis of the neural correlates of social cognition. PloS One. 2011;6(10):e25322.

19. Di Martino A, Ross K, Uddin LQ, Sklar AB, Castellanos FX, Milham MP. Functional brain correlates of social and nonsocial processes in autism spectrum disorders: an activation likelihood estimation meta-analysis. Biol Psychiatry. 2009;65(1):63-74.

20. Blakemore SJ. The social brain in adolescence. Nat Rev Neurosci. 2008;9(4):267-77.

21. Kilford EJ, Garrett E, Blakemore SJ. The development of social cognition in adolescence: an integrated perspective. Neurosci Biobehav Rev. 2016;70:106-20.

22. Di Martino A, Yan CG, Li Q, Denio E, Castellanos FX, Alaerts K, et al. The autism brain imaging data exchange: towards a large-scale evaluation of the intrinsic brain architecture in autism. Mol Psychiatry. 2014;19(6):659-67.

23. Di Martino A, O'Connor D, Chen B, Alaerts K, Anderson JS, Assaf M, et al. Enhancing studies of the connectome in autism using the autism brain imaging data exchange II. Sci Data. 2017:4:170010.

24. Loth E, Charman T, Mason L, Tillmann J, Jones EJH, Wooldridge C, et al. The EU-AIMS Longitudinal European Autism Project (LEAP): design and methodologies to identify and validate stratification biomarkers for autism spectrum disorders. Mol Autism. 2017:8:24

25. Button KS, loannidis JP, Mokrysz C, Nosek BA, Flint J, Robinson ES, et al. Power failure: why small sample size undermines the reliability of neuroscience. Nat Rev Neurosci. 2013;14(5):365-76.

26. Abell F, Happé F, Frith U. Do triangles play tricks? Attribution of mental states to animated shapes in normal and abnormal development. Cognitive Development. 2000;15:1-16.

27. White SJ, Coniston D, Rogers R, Frith U. Developing the Frith-Happe animations: a quick and objective test of Theory of Mind for adults with autism. Autism Res. 2011;4(2):149-54.

28. Moessnang C, Otto K, Bilek E, Schafer A, Baumeister S, Hohmann S, et al. Differential responses of the dorsomedial prefrontal cortex and right posterior superior temporal sulcus to spontaneous mentalizing. Hum Brain Mapp. 2017;38(8):3791-803

29. Moessnang C, Schafer A, Bilek E, Roux P, Otto K, Baumeister S, et al. Specificity, reliability and sensitivity of social brain responses during spontaneous mentalizing. Soc Cogn Affect Neurosci. 2016.

30. Association AP. Diagnostic and statistical manual of mental disorders. In: Association AP, editor. 4 ed. Washington, DC 1994.

31. Association AP. Diagnostic and statistical manual of mental disorders. In: Association AP, editor. 4 ed. Washington, DC. 2000.

32. Association AP. Diagnostic and statistical manual of mental disorders. 5th ed. Arlington: Author; 2013.
33. Organization WH. The ICD-10 Classification of mental and behavioural disorders: diagnostic criteria for research. In: WHO, editor. Geneva. 1993.

34. Charman T, Gotham K. Measurement issues: screening and diagnostic instruments for autism spectrum disorders - lessons from research and practice. Child Adolesc Ment Health. 2013;18(1):52-63.

35. Lord C, Risi S, Lambrecht L, Cook EH Jr, Leventhal BL, DiLavore PC, et al. The autism diagnostic observation schedule-generic: a standard measure of social and communication deficits associated with the spectrum of autism. Jutism Dev Disord. 2000;30(3):205-23.

36. Rutter M, Le Couteur A, Lord C. Autism diagnostic interview-revised. Los Angeles: Western Psychological Services; 2003.

37. Charman T, Loth E, Tillmann J, Crawley D, Wooldridge C, Goyard D, et al. The EU-AIMS Longitudinal European Autism Project (LEAP): clinical characterisation. Mol Autism. 2017:8:27.

38. Jenkinson M, Bannister $\mathrm{P}$, Brady M, Smith S. Improved optimization for the robust and accurate linear registration and motion correction of brain images. Neuroimage. 2002;17(2):825-41.

39. Hus V, Gotham K, Lord C. Standardizing ADOS domain scores: separating severity of social affect and restricted and repetitive behaviors. J Autism Dev Disord. 2014:44(10):2400-12.

40. Constantino J, Gruber C. Social responsiveness scale. In: Volkmar FR, editor. Encyclopedia of Autism Spectrum Disorders. New York: Springer; 2013.

41. Goodman A, Heiervang E, Collishaw S, Goodman R. The 'DAWBA bands' as an ordered-categorical measure of child mental health: description and validation in British and Norwegian samples. Soc Psychiatry Psychiatr Epidemiol. 2011:46(6):521-32.

42. Faul F, Erdfelder E, Lang AG, Buchner A. G*Power 3: a flexible statistical power analysis program for the social, behavioral, and biomedical sciences. Behav Res Methods. 2007;39(2):175-91.

43. Cohen J. Statistical power analysis for the behavioral sciences. Revised Edition. Hillsdale: Erlbaum; 1988.

44. Cremers HR, Wager TD, Yarkoni T. The relation between statistical power and inference in fMRI. PloS One. 2017;12(11):e0184923.

45. Castelli F, Happe F, Frith U, Frith C. Movement and mind: a functional imaging study of perception and interpretation of complex intentional movement patterns. Neuroimage. 2000:12(3):314-25.

46. Moriguchi Y, Ohnishi T, Mori T, Matsuda H, Komaki G. Changes of brain activity in the neural substrates for theory of mind during childhood and adolescence. Psychiatry Clin Neurosci. 2007;61(4):355-63.

47. Tzourio-Mazoyer N, Landeau B, Papathanassiou D, Crivello F, Etard O, Delcroix N, et al. Automated anatomical labeling of activations in SPM using a macroscopic anatomical parcellation of the MNI MRI single-subject brain. Neuroimage. 2002;15(1):273-89.

48. Eickhoff SB, Heim S, Zilles K, Amunts K. Testing anatomically specified hypotheses in functional imaging using cytoarchitectonic maps. Neuroimage. 2006;32(2):570-82

49. Wang AT, Lee SS, Sigman M, Dapretto M. Neural basis of irony comprehension in children with autism: the role of prosody and context. Brain. 2006;129(Pt 4):932-43.

50. Sebastian CL, Fontaine NM, Bird G, Blakemore SJ, Brito SA, McCrory EJ, et al. Neural processing associated with cognitive and affective Theory of Mind in adolescents and adults. Soc Cogn Affect Neurosci. 2012;7(1):53-63.

51. Moor BG, Macks ZA, Guroglu B, Rombouts SA, Molen MW, Crone EA. Neurodevelopmental changes of reading the mind in the eyes. Soc Cogn Affect Neurosci. 2012;7(1):44-52.

52. Poldrack RA. Interpreting developmental changes in neuroimaging signals Hum Brain Mapp. 2010;31(6):872-8

53. Mills KL, Lalonde F, Clasen LS, Giedd JN, Blakemore SJ. Developmental changes in the structure of the social brain in late childhood and adolescence. Soc Cogn Affect Neurosci. 2014;9(1):123-31.

54. Ioannidis JP. Why most published research findings are false. PLoS Med 2005:2(8):e124.

55. loannidis JP. Excess significance bias in the literature on brain volume abnormalities. Arch Gen Psychiatry. 2011;68(8):773-80.

56. Halsey LG, Curran-Everett D, Vowler SL, Drummond GB. The fickle P value generates irreproducible results. Nat Methods. 2015;12(3):179-85.

57. Poldrack RA, Baker Cl, Durnez J, Gorgolewski KJ, Matthews PM, Munafo MR, et al. Scanning the horizon: towards transparent and reproducible neuroimaging research. Nat Rev Neurosci. 2017;18(2):115-26.

58. Apperly IA, Butterfill SA. Do humans have two systems to track beliefs and belief-like states? Psychol Rev. 2009;116(4):953-70. 
59. Grossmann T. The development of social brain functions in infancy. Psychol Bull. 2015;141(6):1266-87.

60. Kulke L, von Duhn B, Schneider D, Rakoczy H. Is implicit theory of mind a real and robust phenomenon? Results from a systematic replication study. Psychol Sci. 2018;29(6):888-900.

61. Richardson H, Lisandrelli G, Riobueno-Naylor A, Saxe R. Development of the social brain from age three to twelve years. Nat Commun. 2018;9(1):1027.

62. McCormick EM, van Hoorn J, Cohen JR, Telzer EH. Functional connectivity in the social brain across childhood and adolescence. Soc Cogn Affect Neurosci. 2018;13(8):819-30.

63. Fox MD, Corbetta M, Snyder AZ, Vincent JL, Raichle ME. Spontaneous neuronal activity distinguishes human dorsal and ventral attention systems. Proc Natl Acad Sci U S A. 2006;103(26):10046-51.

64. Apperly IA, Samson D, Humphreys GW. Domain-specificity and theory of mind: evaluating neuropsychological evidence. Trends Cogn Sci. 2005;9(12):572-7.

65. Lombardo MV, Lai MC, Baron-Cohen S. Big data approaches to decomposing heterogeneity across the autism spectrum. Mol Psychiatry. 2019.

66. Glover GH, Mueller BA, Turner JA, van Erp TG, Liu TT, Greve DN, et al. Function biomedical informatics research network recommendations for prospective multicenter functional MRI studies. J Magn Reson Imaging. 2012;36(1):39-54.

67. Wang AT, Lee SS, Sigman M, Dapretto M. Reading affect in the face and voice: neural correlates of interpreting communicative intent in children and adolescents with autism spectrum disorders. Arch Gen Psychiatry. 2007; 64(6):698-708.

68. Lai MC, Lombardo MV, Chakrabarti B, Ruigrok AN, Bullmore ET, Suckling J, et al. Neural self-representation in autistic women and association with 'compensatory camouflaging'. Autism. 2018;1362361318807159.

69. Hahamy A, Behrmann M, Malach R. The idiosyncratic brain: distortion of spontaneous connectivity patterns in autism spectrum disorder. Nat Neurosci. 2015;18(2):302-9.

70. Muller RA, Shih P, Keehn B, Deyoe JR, Leyden KM, Shukla DK. Underconnected, but how? A survey of functional connectivity MRI studies in autism spectrum disorders. Cerebral Cortex. 2011;21(10):2233-43.

71. Thye MD, Bednarz HM, Herringshaw AJ, Sartin EB, Kana RK. The impact of atypical sensory processing on social impairments in autism spectrum disorder. Dev Cogn Neurosci. 2018:29:151-67.

72. Happe F, Cook JL, Bird G. The structure of social cognition: in(ter)dependence of sociocognitive processes. Ann Rev Psychol. 2017;68: 243-67.

73. Clements CC, Zoltowski AR, Yankowitz LD, Yerys BE, Schultz RT, Herrington JD. Evaluation of the social motivation hypothesis of autism: a systematic review and meta-analysis. JAMA Psychiatry. 2018;75(8):797-808.

74. Kinnaird E, Stewart C, Tchanturia K. Investigating alexithymia in autism: a systematic review and meta-analysis. Eur Psychiatry. 2019;55:80-9.

\section{Publisher's Note}

Springer Nature remains neutral with regard to jurisdictional claims in published maps and institutional affiliations.

Ready to submit your research? Choose BMC and benefit from:

- fast, convenient online submission

- thorough peer review by experienced researchers in your field

- rapid publication on acceptance

- support for research data, including large and complex data types

- gold Open Access which fosters wider collaboration and increased citations

- maximum visibility for your research: over $100 \mathrm{M}$ website views per year

At $\mathrm{BMC}$, research is always in progress.

Learn more biomedcentral.com/submissions 